\title{
Total hip arthroplasty results in the osteoarthritis secondary to developmental dyspla- sia of the hip (Crowe type III and IV)
}

\author{
Bülent Köksal*, N. Turgut Karaismailoğlu, Ali Terkuran, E. Eren Desteli, B. Özgür Yazıcı, Hicabi Sezgin, Fatih Yanık \\ Department of Orthopaedics and Traumatology, Medical Faculty, Ondokuz Mayls University, Samsun, Turkey
}

\begin{tabular}{|c|c|}
\hline \multicolumn{2}{|c|}{ ARTICLE INFO } \\
\hline \multicolumn{2}{|c|}{ Article History } \\
\hline Received & 09 / 01 / 2012 \\
\hline Accepted & $19 / 01 / 2012$ \\
\hline
\end{tabular}

\section{* Correspondence to}

Bülent Köksal

Department of Orthopaedics

And Traumatology,

Medical Faculty,

Ondokuz Mayıs University,

Samsun, Turkey

e-mail: bulentkoksal19@hotmail.com

\section{Keywords:}

Developmental hip dysplasia

Arthroplasty

Osteoarthritis

Femoral shortening osteotomy

Trochanteric osteotomy

\section{ABSTRACT}

Together with standard total hip arthroplasty (THA), total hip arthroplasty in hip dysplasia and hip dislocation cases has also increased in our country. In this study short and long term evaluation of total hip arthroplasty cases secondary to osteoarthritis following Crowe type III and IV developmental dysplasia of the hip (DDH) has been evaluated. In Ondokuz Mayis University Medical Faculty Department of Orthopaedics and Traumatology, we have retrospectively evaluated 78 THA patients presenting with high dislocation between May 2000 and May 2008. Out of 65 patients seventy-eight hips were assessed in the study. One of the patients was male and remaining 64 patients were females. Thirtyfour (43.6\%) patients were operated from their right hips, while $44(56.4 \%)$ patients were operated from their left hips. Mean follow up time was 49.5 months (10 - 105 months). Seventy-eight hips were operated using posterolateral approach. Patients were postoperatively evaluated in 1st, 3rd, 5th, 6th and 12th months and once for the subsequent year. Clinical Harris scale and radiological Callaghan and Engh criteria were used to evaluate the results. Results were evaluated both clinically and radiologically. Twenty-two (28.2\%) of the 78 hips were determined to be Crowe type III and $56(71.8 \%)$ of them were type IV. Mean value of limb length deficiency before operation was $3.29 \mathrm{~cm}$ (distribution $0-7.5 \mathrm{~cm}, \pm 2.06$ ), in the final control it was determined to be $0.84 \mathrm{~cm}$ (distribution $0-2$ $\mathrm{cm}, \pm 0.89)$. Trochanteric osteotomy was performed for $41(52.5 \%)$ hips and sub trochanteric osteotomy was performed for $21(\% 26.9)$ hips. In these osteotomized hips acetabulum was moved to its plausible location. Shortening was not performed in $16(20.5 \%)$ patients. Mean value for distance of greater trochanter - minor trochanter was $49.20 \mathrm{~mm}$. $(31-65 \mathrm{~mm}, \pm 6.5)$. In $21(26.9 \%)$ of the patients autograft taken from femoral head was put on to superolateral of acetabulum. Acetabular covering was 20-35\%, and the median value was $30(20-35) \%$. In $10(12.8 \%)$ of the 62 osteotomized hips non-union was detected. In 7 patients $(8.9 \%)$ there was late dislocation (except one). Preoperative mean Harris score was $41.9( \pm 8.9)$ while postoperative mean Harris score was $88.9( \pm 9.5)$. According to these results, $69(69.7 \%)$ of the cases were excellent, $22(22.2 \%)$ were very good, $6(6.1 \%)$ were good and $2(2 \%)$ were average. Results of total hip arthroplasties following developmental dysplasia or dislocation of hip are quite good. When examining the complaints leading to losses in PO Harris score, it has been found out that holding the handrails while climbing up the stairs as well as wearing shoes and socks have been detected to be the factors resulting in most decreases in the score. On the other hand, it has apparently been showed that in all the criteria including pain, walking, activity, deformity and latitude of motion which are the general basis of Harris evaluation schedule, point increase that is recovery has been determined in PO period. J. Exp. Clin. Med., 2012; 29:33-37

(C) 2012 OMU All rights reserved

\section{Introduction}

Developmental dysplasia of the hip (DDH) is one of the most substantial underlying reasons of secondary formation of osteoarthritis. Due to proximal displacement of femur, flexion and adduction deformity of the hip, valgus deformity of the knee, the extremity becomes shortened. Compatible with this, functional scoliosis arising from this deformity becomes structural. Posterior ascending of femoral head results in pelvic curvature thereby leading to proliferation of lumbar lordosis. Due to the development of osteoarthritis, hip pain even occurring while at rest and increasing owing to activity develops.

Due to the fact that the implants used in the treatment of coxarthrosis have been improved, and that experiences have 
been increased and that long term results have become hopeful, hip arthroplasty has recently become one of the most used treatment methods.

The objective of this study is to evaluate the results of total hip arthroplasty that we applied to the patients presenting with Crowe Type III and Type IV hip dysplasia between 2000 and 2008.

\section{Materials and methods}

In Ondokuz Mayis University Medical Faculty Department of Orthopaedics and Traumatology, we have retrospectively evaluated 78 THA patients presenting with high dislocation between May 2000 and May 2008. Out of 65 patients, seventy-eight hips were assessed in the study. All these patients were operated by the same surgeon using posterolateral incision. The clinical and radiological assessments of the patients were performed by the same physician.

Radiological assessment was performed with the early and late postoperative direct graphs. Acetabular component angle, femoral component status, location of acetabular component, modifications in components, osteotomy method and acetabular roof graft were evaluated. In the clinical evaluation of the patients, preoperative and postoperative length differences were measured with trendelenburg test and Harris hip score.

\section{Surgical technique}

While patient were in bed at supine position, the hip adductors were examined in the hip abduction. Only if the adductor contracture was detected, adductor tenotomy was performed by intervening through some points with fine ended scalpel. Following this intervention, no suture materials were used and solely compression was sufficient in scar. The patient was laid on the side in a fashion that the hip to be operated would be up. Posterolateral incision was performed in the hip. Joint capsule and soft tissues in the proximal femur were loosened. On the condition that femur head descend to the real acetabulum only when subtracting the distance difference between trochanter major and minor, trochanter major osteotomy was performed in a pattern involving the proximal of the muscle without lacerating the rectus femoris muscle. Initially, the acetabulum was prepared then femoral canal was prepared. If the hip were not easily reduced, intertrochanteric femoral shortening osteotomy was performed up to trochanter minor grade and femoral stem was inserted. One or two wire cables were passed through the proximal femur and the hip was reduced. Trochanter major - enabling the sufficient gluteus medius tense - was fixed to the proximal femur with wire cable (Fig. 1).

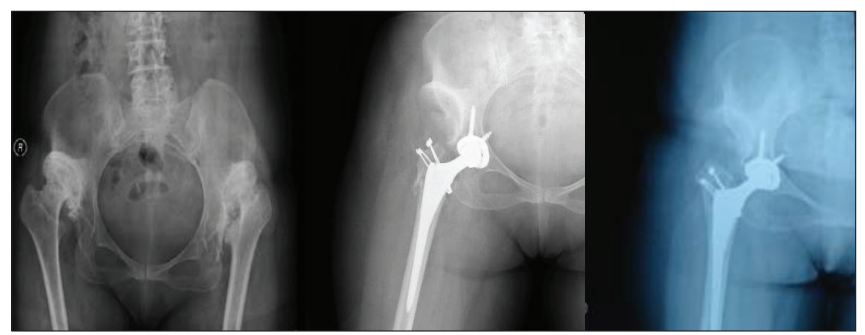

Fig. 1. FPreoperative AP right hip graph PO AP right hip graph PO $12^{\text {th }}$ month AP left hip graph
In addition, it was resolved that subtrochanteric osteotomy would be performed by considering the fact that femoral head was located in a high level and that trochanteric osteotomy would not become effective enough. Firstly, the small sized carvers were used to open the femoral medulla and oblique osteotomy through subtrochanteric region was performed and the femoral shortening which was necessary for the reduction of femoral head was performed through subtrochanteric region. Later, acetabular cup was inserted following the preparation of acetabulum. Following the insertion of femoral stem, the hip was reduced. No additional fixation materials except stem were applied to the femoral osteotomy region (Fig. 2).

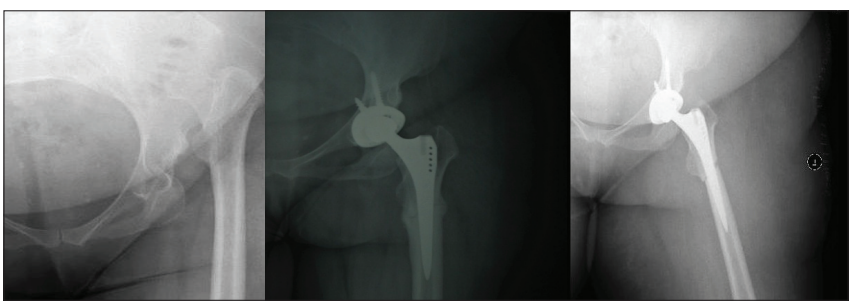

Fig. 2. Preoperative AP left hip graph PO AP left hip graph PO $10^{\text {th }}$ month AP left hip graph

Square sectional femoral stem was particularly used. The patient stood on the postoperative $3^{\text {rd }}$ day.

\section{Statistical analysis}

The findings and results acquired from the study were analyzed with SPSS 16.0 package software. The results were defined with average \pm standard deviation and number (\%). Normal distribution test (Shapiro Wilk Test) was used to perform the statistical comparisons of the groups. Due to the fact that the data acquired were not complying with the normal distribution, they were compared with Mann-Whitney U test - a non-parametric test. Statistical significance level was accepted to be $\mathrm{p}<0.05$.

\section{Results}

Sixty-five patients including one male and 64 female subject to total hip arthroplasty applied to the coxarthrosis in the ground of developmental hip dysplasia were involved in our study. Seventy-eight hips of these 65 patients were evaluated. The mean age of the patients was $43.6 \pm 9.06$ years. Out of these seventy-eight hips $34(43.6 \%)$ were right while 44 $(56.4 \%)$ were left. Of the patients, $22(28.2 \%)$ were Crowe Type III while remaining 56 (71.8\%) were Crowe Type IV.

The mean value of preoperative length was calculated to be $3.29 \pm 2.06 \mathrm{~cm}^{2}$. The length difference in the PO period was measured as $0.84 \pm 0.89 \mathrm{~cm}^{2}$. Preoperative trendelenburg test results were positive in $61(78.2 \%)$ patients and negative in $17(21.8 \%)$ patients. Postoperative trendelenburg test results were positive in six $(7.7 \%)$ patients and negative in 72 $(92.3 \%)$ patients.

The patients were clinically evaluated according to their preoperative and postoperative Harris hip score results. Mean value of Harris hip score of 22 hips of Crowe Type III was $44(32-54)$ as preoperative but $89(56-98)$ as postoperative. Mean value of Harris hip score of 58 hips of Crowe Type IV was 38 (27-68) as preoperative but 91.5 (65-100) as postoperative. 
In the radiographic controls performed in the early postoperative stage, mean value of acetabular component angle was detected to be $48^{\circ}\left(34^{\circ}-64^{\circ}\right)$. Within this duration up to the final control, cup angles were determined to be modified in six patients as $1-12^{\circ}$ (mean $4.5^{\circ}$ ). It was determined that five $(5.05 \%)$ patients presented with acetabular cup migration, two $(2.02 \%)$ with horizontal, one $(1.01 \%)$ with both vertical and horizontal displacement. While the vertical migration was $2 \mathrm{~mm}$, horizontal migration was measured as 1-3 $\mathrm{mm}$ (mean $2.3 \mathrm{~mm}$ ). When assessing the radiolucent lines, De Lee and Charnley were used for acetabulum and Gruen systems were used for femoral side. Accordingly, in zone III $1 \mathrm{~mm}$ sized asymptomatic radiolucent streaking was detected in 2 acetabulums. On the other hand, in the femoral side, in four of the patients in several zones, asymptomatic radiolucent sites were detected. Such patients were clinically stabilized.

In 21 patients (26.9\%) undergoing acetabular graft due to the insufficiency of superior wall, auto graft taken from femoral head was inserted in the superolateral of acetabulum. The covering percentage of graft on acetabular graft was detected to be $20-35 \%$ and mean value was calculated to be $30 \%$ (20-35). Of these patients 11 were Crowe Type IV, 10 were Crowe Type III. For graft fixation, single titanium screw was used in seven hips, while two titanium screws in 12 hips and three in two hips were used. It was observed that full union was developed in 6 months in average in 15 hips, and that in $7 \%(5-20 \%)$ of the acetabular component in 6 hips in average resorption was observed. Even in the hips with $20 \%$ graft resorption, due to the fact that acetabulum covering percentage was more than $90 \%$, in none of the patients acetabular loosening was detected.

In the preoperative evaluation, the preoperative distance between trochanter major and minor was measured as 49.2 $( \pm 6.5) \mathrm{mm}$. In 41 patients $(52.5 \%)$ with sufficient distance for shortening 6-34 $\mathrm{mm}$ and in average $21.6( \pm 5.8) \mathrm{mm}$ shortening was performed. 21 patients $(26.9 \%)$ were exposed to subtrocantheric osteotomy and 40-60 $\mathrm{mm}$ in average $47( \pm 6.2)$ mm shortening was performed. In $16(20.5 \%)$ hips, no shortening procedures were applied. In 72 patients $(92.3 \%)$, it was inserted in the actual acetabulum and in 6 patients $(7.7 \%)$ in a location between actual and pseudo acetabulum.

In two $(4.8 \%)$ out of 41 hips exposed to trochanteric osteotomy, delayed union (in the month 8) and non-union in 9 hips $(21.9 \%)$ was detected. In one of these patients presenting with pain and walking disorder, repeated surgical operation was performed on the non-union line. Despite the fact that fixation was performed with curettage + grephonage +1 cuff +2 cables, no union was acquired on the non-union line. As the complaints of the patient reduced, no additional interventional procedures were performed. Mean Harris hip score of such patients in the final control was determined to be 82.9. In all the remaining $30(73.2 \%)$ hips, osteotomy region was radiologically unified at six month. In one of the 21 hips exposed to subtrohcanteric osteotomy, non-union was detected. Due to the fact that delayed union was detected in one patient, grenophage +4 holed plaque fixations were performed in the month 10 . No problems were detected during the follow up and the union was fully performed.

\section{Discussion}

In the hip osteoarthritis secondary to developmental hip dysplasia, total hip prosthesis application is quite more different than total hip proshesis due to primary osteoarthritis. The anatomic and biomechanical modifications occurring due to the proximal migration of femur and in bone and soft tissues strengthen the arthroplasty in technical pattern and make them more susceptible to complication (Symeonides et al., 1997).

The objective of total hip arthroplasty in patients with developmental hip dysplasia is to acquire a stabilized, long life, painless and functional hip joint. In the femur and acetabulum, bone-like modifications secondary to dysplasia and the eradication of pathologies in the soft tissues as well as the differences in practices lead to controversies. No clear consensus was available in the literature from the incision type to acetabulum reconstruction, to shortening size in the femoral intervention, and to prosthesis type to be used (Haddad et al., 1999; Ito et al., 2003; Hartofilakidis and Karachalios, 2004; Eskelinen et al., 2005; Klapach, 2005; Hampton and Harris, 2006).

In the subluxated and dislocated hips, in the arthroplasty application, one of the technically complex aspects is the difficulty in acquiring a healthy bone tissue and stabilized and long life acetabular component. The site in which the acetabular bone support is mostly seen is the actual acetabulum or the peripheral zone. We descended the acetabulum to the actual zone and rimerized it up to the medial cortex. We inserted the plausible and small sized acetabular component to this zone. Despite the procedure applied, we supported the acetabulum roof with the grafts that prepared from the femur head in the patients with acetabular failure. We did not find any loosening in these patients. The use of acetabular component in the structural bone grafts was introduced by Dunn and Hess and Harris by using femur head as autograft like a support in the cemented acetabular components (Dunn and Hess, 1976; Mulroy and Jr Harris, 1990). Despite the fact that early graft union was detected in Harris series, at the end of the 7 th year; $20 \%$, and at the end of the 11th year; $46 \%$ of loosening was detected (Mulroy and Jr Harris, 1990). The fact that such poor results acquired in long term studies were associated with the use of femur head exposed to sclerotic and cystic degeneration and that the graft covered more than $40 \%$ of the acetabular component.

In the porous coated without cement acetabular components, with the insertion of live bone tissue between the porous surfaces a biological fixation takes place and thereby reducing the load on graft and decreasing the possibility of mechanical insufficiency of the graft. It was detected that femur head was unified to auto graft acetabulum at 7 th month, and that remodelation was completed at 18th month, (Hasegawa et al., 1996). No loosening, migration and pelvic osteolysis were detected in the 8th year results of 24 hips exposed to uncemented acetabular component used by Anderson and Harris.

Linde, in a 15 year comparative study, reported that loosening ratio in the acetabular components with the actual acetabulum location was $13 \%$ and $42 \%$ in the high locations. In the cases when the acetabular component was not inserted in the actual acetabulum, the modifications in the rotation 
center of the hip led to substantial modifications in the hip biomechanics thereby negatively affecting the reconstruction resistance (Johnston et al., 1979; Flecher et al., 2008). In our 49.5 months follow up, in $1 \%$ of the patients, loosening in the actual site of acetabular component was detected. We considered that the most substantial factor of such low grade was associated with the reduction of acetabular component to the best place of bone stock.

High hip dislocation (Crowe Type III and Type IV) was accompanied by pathological bone-like modifications in proximal femur as well as peripheral soft tissue modifications in the secondary hip osteoarthritis (Charnley and Feagin, 1973; Crowe et al., 1979; Mendes, 1981; Sugano et al., 1998; Haddad et al., 1999). In the cases with the location of acetabular component in the actual acetabulum thereby requiring the descend of the femur to the distal site. If the secondary soft tissues were not intervened plausibly, numerous complications could be detected. In such cases, abductor muscles, iliopsoas, rectus femoris, gluteus maximus, fascia lata and other femoral muscles were shortened and the tensile was increased. Priformis was shortened and other external rotators were extended. The fact that sufficient function was detected in the abductor muscles particularly following the arthroplasty requires for the descend of femur to the distal for the recovery of Trendelenburg hitch and equalization of extremity length single sided dislocations or reduction of the difference (Dunn and Hess, 1976; Gorski, 1988; Holtgrewe and Hungerford, 1989; Paavilainen et al., 1990; Paavilainen et al., 1993; Paavialinen, 1997; Symeonides et al., 1997; Yasgur et al., 1997; Hartofilakidis et al., 1998). Therefore as in the total knee prosthesis application, establishment of soft tissue balance is of great importance. In order for loosening the soft tissue, particularly adductor and illiopsoas tenomyopathy should be performed, however, the gluteus maximus tendon may be lacerated from the femur adhesion in the cases presenting with no reductions and the adductor muscles may be abraded from the linea aspera bluntly (Mattingly, 2006).

Following the application of anaesthesia, during the adductor tendon examination performed while in supine position, in order for facilitating the descend of the femur to distal of 60 hips $(76.9 \%)$ with tensed adductor tendon and abduction restriction were exposed to percutaneous adductor tenotomy. During the descend of the femur to distal, 2 hips (2.5\%) with extreme tensile, tensor fascia latace was loosened.

In the literature there are some studies claiming that in the patients undergoing trochanteric osteotomy and during the surgical procedure, in addition to the lateral and distal ascend of the trochanter, exposure is better and the rimerization of the femoral canal is easier and prosthesis components may be easily and correctly inserted (Dunn and Hess, 1976; Mendes, 1981; Paavilainen et al., 1990). Moreover, there are some disadvantages such as the bone loss of the trochanteric osteotomy in the proximal femur, poor provision of rotational and axial stability, non-union of trochanter and bursit formation, as well.

We-with the trochanteric osteotomy-aimed to major lateralization, distal transportation and femur shortening rather than acetabulum exposure. In two hips out of forty one hips delayed union (in the month 8) was detected and in 9 hips $(21.9 \%)$ non-union was formed. In all the remaining 30 hips $(73.2 \%)$, the osteotomy zone was unified at six month. Iida et al. in their series including 133 hips detected that non-union up to $19 \%$ was found (Iida et al., 2000). In solely 2 of the cases $(6.6 \%)$ out of 30 hips fixated with 2 cables, and in the treatment of $7(63.6 \%)$ of the 11 patients exposed to single cable or two wires, non-union disorder was detected in our study. We consider that the most substantial reason of nonunion formation is associated with non-rigid wire or single cable fixation. In addition, we also think that in the patients subject to trochanteric osteotomy with rigid fixation have better union results and earlier mobilization may be acquired prior to the union of osteotomy line.

Without applying trochanter major osteotomy, the subtrochanteric osteotomy may be applied in three patterns as: oblique, straight and stepped. While plaque and screw fixation have been recommended for the rotational stability in the straight osteotomy line, it is not necessary for the stepped and oblique osteotomy. In our study, 21 out of 78 hips were subject to oblique and 1 was subject to straight femoral subtrochanteric shortening. In all the hips Crowe Type IV dislocation was detected. Our mean resection amount was measured as $47 \mathrm{~mm}$ (distribution 40-60 $\mathrm{mm} \pm 6.2$ ). Uncemented femoral component was preferred in all the hips. We did not perform plaque fixation in the oblique osteotomy but we placed the grafts acquired from femur head in order for facilitating the union of osteotomy line.

We frequently applied subtrochanteric osteotomies in the patients with the requirement of descend of five $\mathrm{cm}$ or more of the hip rotation center.

Dislocation following the total hip prosthesis is a serious complication for both patient and surgeon. Prevalence has been reported to be $1-10 \%$. Eight PO dislocations in seven patients in our study were detected and patients were exposed to closed reduction and for two weeks under derogation cast it was followed. In three patients linear 20 degree was replaced and placed again in one patient following the recovery of the anterversion of femoral stem. We consider that weakness in the posterior components due to posterolateral incision and use of small cup lead to the increase of dislocation susceptibility despite the fact that this dislocation ratio is within the borders of literature ratios.

On the other hand, it has apparently been showed that in all the criteria including pain, walking, activity, deformity and latitude of motion which are the general basis of Harris evaluation schedule, point increase that is recovery has been determined in PO period. When examining the complaints leading to losses in PO Harris score, it has been found out that holding the handrails while climbing up the stairs as well as wearing shoes and socks have been detected to be the factors resulting in most decreases in the score. As seen, the results of THP are very satisfactory in the patients with hip dislocation with the correct method and by an experienced surgeon as well as in the surgical treatment of pain not responding to conservative treatment and with functional loss.

\section{REFERENCES}

Chougle, A., Hemmady, M.V., Hodgkinson, J.P., 2006. Long-term survival of the acetabular component after total hip arthroplasty with cement in patients with developmental dysplasia of the hip. J. Bone Joint Surg. 88, 71-79.

Crowe, J.F., Mani, J., Ranawat, C., 1979. Total hip replacement in congenital dislocation and dysplasia of the hip. J. Bone Joint Surg. 61, 15-23. 
Dunn, H.K., Hess, W.E., 1976.Total hip reconstruction in chronically dislocated hips. J. Bone Joint Surg. 58, 838-845.

Eskelinen, A., Helenius, I., Remes, V., Ylinen, P., Tallroth, K., Paavilainen, T., 2005.Cementless total hip arthroplasty in patients with high congenital hip dislocation. J. Bone Joint Surg. 88, 80-91.

Flecher, X., Parratte, S., Brassart, N., Aubaniac, J.M., Argenson, J.N., 2008.Evaluation of the hip center in total hip arthroplasty for old developmental dysplasia. J. Arthroplasty. 8, 1189-1196.

Gorski, J.M., 1988. Modular no cemented total hip arthroplasty for congenital dislocation of the hip. A case report and design rationale. Clin. Orthop. 228, 110-116.

Haddad, F.S., Masri, B.A., Garbuz, D.S., Duncan, C.P., 1999. Primary total replacement of the dysplastic hip. AAOS Instruct Course LECT. J. Bone Joint Surg. 81, 1462-1482.

Hampton, B.J., Harris, W.H., 2006. Primary cement less acetabular components in hip with severe developmental dysplasia or total dislocation. A concise follow-up, at an average of sixteen years, of a previous report. J. Bone Joint Surg. 88, 1549-1552.

Harris, W.H., 1988. Total hip arthroplasty in the management of the congenital hip dislocation. In Callaghan, J.J., Rosenberg, A.G., Rubash, H.E., (eds): The adult hip. Lippincott-Rawen, Philadelphia.1651-1682

Hartofilakidis, G., Karachalios, T., 2004. Total hip arthroplasty for congenital hip disease. J. Bone Joint Surg. 86, $242-250$.

Hartofilakidis, G., Stamos, K., Karachalios, T., 1998. Treatment of high dislocation of the hip in adults with total hip arthroplasty. J. Bone Joint Surg. 80, 510-517.

Hasegawa, Y., Iwata, H., Iwase, T., Kawamoto, K., Iwasada, S., 1996. Cement less total hip arthroplasty with autologous bone grafting for hip dysplasia. Clin. Orthop. 324, 179-186.

Holtgrewe, J.L., Hungerford, D.S., 1989. Primary and revision total hip replacement without cement and with associated femoral osteotomy. J. Bone Joint Surg. 71, 1487-1495.

Iida, H., Matsusue, Y., Kawanabe, K., Okumura H, Yamamuro T, Nakamura T., 2000. Cemented hip arthroplasty with acetabular bone graft for developmental dysplasia. J. Bone Joint Surg. 82, 176-184.

Ito H, Matsuno T, Minami A, Aoki Y; 2003 Intermediate-term results after hybrid total hip arthroplasty for the treatment of dysplastic hips. J Bone Joint Surg.85-A: 1725-1732.

Johnston, R.C., Brand, R.A., Crowninshield, R.D., 1979. Recostruction of the hip. A mathematical approach to determine optimum geometric relationships. J. Bone Joint Surg. 61, 639-652.

Klapach, A.S., Callaghan, J.J., Miller, K.A., Goetz, D.D., Sullivan, P.M., Pedersen, D.R., Johnston, R.C., 2005. Total hip arthroplasty with cement and without acetabular bone graft for severe hip dysplasia. A concise follow-up, at a minimum of twenty years, of a previous report. J. Bone Joint Surg. 87, 280-285.

Mattingly DA; 2006 The modular stem in developmental dysplasia of the hip. In Barrack RL, Rosenberg AG (eds): Master techniques in orthopaedic surgery. The Hip. Lippincott Williams, Philadelphia. 249-260.

Mendes, D.G., 1981. Total hip arthroplasty in congenital dislocated hips. Clin. Orthop. 161, 163-179.

Mulroy, R.D., Jr Harris, W.H., 1990. Failure of acetabuler autografts in total hip arthroplasty. Increasing incidence: A follow-up note. J. Bone Joint Surg.72, 1536-1540.

Paavialinen, T., 1997. Total hip replacement for developmental dysplasia of the hip. Acta. Orthop. Scand. 68, 77-84.

Paavilainen, T., Hoikka, V., Paavolainen, P., 1993. Cement less total hip arthroplasty for congenitally dislocated or dysplastic hips. Clin. Orthop. 297, 71-81.

Paavilainen, T., Hoikka, V., Solonen, K.A., 1990. Cement less total replacement for severely dysplastic or dislocated hips. J. Bone Joint Surg. 72, 205-211.

Sugano, N., Noble, P.C., Kamaric, E., Salama, J.K., Ochi, T., Tullos, H.S., 1998. The morphology of the femur in developmental dysplasia of the hip. J. Bone Joint Surg. 81, 711-719.

Symeonides, P.P., Pournaras, J.J., Petsatodes, G., Christoforides, J., Hatzokos, I., Pantazis, E., 1997. Total hip arthroplasty in neglected congenital dislocation of the hip. Clin. Orthop. 341, 51-55.

Yasgur, D.J., Stuchin, S.A., Adler, E.M., DiCesare, P.E., 1997. Subtrochanterik femoral shortening osteotomy in total hip arthroplasty for hiriding developmental dislocation of the hip. J. Arthroplasty. 12, 880-888. 